\title{
DIFFERENTIALLY GENE EXPRESSION IN THE BRAIN OF COMMON CARP (CYPRINUS CARPIO) RESPONSE TO COLD ACCLIMATION
}

\author{
Liqun Liang ${ }^{1,2, *}$, Shaowu $\mathrm{Li}^{2,3}$, Yumei Chang ${ }^{2}$, Yong $\mathrm{Li}^{2}$, Xiaowen $\mathrm{Sun}^{2}$, \\ Qingquan Lei ${ }^{1}$ \\ ${ }^{1}$ Harbin University of Science and Technology, Harbin, China, 150080 \\ ${ }^{2}$ Heilongjiang River Fisheries Research Institute, Chinese Academy of Fishery Sciences, \\ Harbin, China, 150070 \\ ${ }^{3}$ College of Life Sciences and technology, Shanghai Fisheries University, Shanghai, China, 200090 \\ * Corresponding author, Address: Heilongjiang River Fisheries Research Institute, Chinese \\ Academy of Fishery Sciences, 43 Songfa Road, Daoli District, Harbin, 150070, P. R. China, \\ Tel: +86-451-84861314, Fax: +86-451-84604803, Email: llq-1019@163.com
}

Abstract: There are a variety of approaches to identify groups of genes that change in expression in response to a particular stimulus or environment. We here describe the application of suppression subtractive hybridization ( $\mathrm{SSH}$ ) for isolation and identification genes in the brain of common carp (Cyprinus carpio) under cold temperatures. The materials were prepared through cooling the hybrid F2 of purse red carp (cold-tolerant strains) and bighead carp (cold-sensitive species) to different regimes of temperatures. A subtracted cDNA library containing 2000 clones was constructed. About 60 positive clones were identified to express differentially by dot blotting in screening 480 clones. Sequencing 26 clones and aligning in GenBank/EMBL database using blastn searching engine, 15 genes showed higher similarities with $85-98 \%$. These annotated genes contained (1) genes for transcription factors and gene products involved in signal transduction pathways such as zinc-finger protein, brevican; (2) genes involved in lipid metabolism such as Acyl-CoA synthetases, and (3) genes involved in the translational machinery such as cytochrome c oxidase, ependymin glycoprotein. In addition, real-time PCR was also conducted to validate these genes. To sum up, we believe this study will make an important contribution to elucidate the possible mechanisms on fish cold tolerance at a molecular level.

Keywords: cold tolerance, suppression subtractive hybridization (SSH), real-time PCR, Cyprinus carpio

Liang, L., Li, S., Chang, Y., Li, Y., Sun, X. and Lei, Q., 2008, in IFIP International Federation for Information Processing, Volume 258; Computer and Computing Technologies in Agriculture, Vol. 1; Daoliang Li; (Boston: Springer), pp. 331-339. 


\section{INTRODUCTION}

Temperature has been recognized as a major environmental factor at the molecular, cellular, tissue, organism and ecosystem levels of biological hierarchy. Low temperature may make the cell threaten by a number of physiological and developmental changes. For aquatic ectotherms, visually observed changes associated with decreased temperature include changes in behavior and coloration. As the decline of temperature, fish exhibits a general decline in activity, respiration capacity, immune capacity and an abrupt loss of equilibrium. In addition, many fish will cease feeding and its muscle become more rigid until to death. It is very necessary to improve the ability of cold tolerance in fish, which may be expected to enlarge the culture areas of some thermophilic fish and improve their disease-resistant ability. It is also important for the study of cold tolerance in all other organisms.

Common carp (Cyprinus carpio) is a major kind of fish in the aquaculture industry around China, which can survive at the temperature ranging from $0 \square$ to $35 \square$. Many documents reported common carp could alter enzyme activities, membrane fluidities and behaviors to adapt to lower temperature. It is reported that lower temperature had an effect on lipid composition of cell membrane (Yeo et al., 1997). With the decline of temperature, fish produce lots of long-chain unsaturated fatty acid, which will promote membrane fluidity of cells. Cossins et al. (2002) also verified the importance of desaturase induction in the inducible cold tolerance of carp. As for the enzyme activities, Brown (1960) brought forth that there are two pathways of glucose metabolism and activities of LDH isoenzyme increased response to cold acclimation in carp liver. Few studies reported the effect of temperature on behavioural decision-making under predation risk. Fraser et al. (1993) found that Atlantic salmon juvenile use low water temperature as a cue to switch from diurnal to nocturnal foraging. At present, many coldinduced genes have been isolated in teleost fish, including cytochrome $c$ oxidase, cytochrome P450, antifreeze protein, methallothionein-1, carnitine palmitoyltransferase I, adenine nucleotide translocase and ependimin (Hardewig et al., 1999; Kloepper-Sams \& Stegeman, 1992; Pickett et al., 1983; Beattie et al., 1996; Rodnick \& Sidell, 1994; Roussel et al., 2000; Tang et al., 1999). The brain is the organ that senses temperature and makes instructions to cold acclimation. It is possible that cold-induced genes in the brain contribute to the control and regulation of the acclimation responses. Besides of the cold-induced alternations in the composition of the phospholipids, the release of neurotransmitters and hormones in the brain has also been reported (Yeo et al., 1997; Tiku et al., 1996; Poli et al., 1997). 
Moreover, studies on the activities of $A c h E$ in brain showed that AchE of some thermo fish becomes unstable at low temperature and possibly exists two isomers at different temperature (Baldwin \& Hochachka, 1997). However, little knowledge are known on the molecular mechanism under temperature acclimation.

In the present study, we set out an experimental fish system and describe the application of suppression subtractive hybridization (SSH) on isolation and identification of the cold tolerance-related genes in the brain of common carp in order to further understand the molecular process involved.

\section{MATERIALS AND METHODS}

\subsection{Construction of experimental fish system}

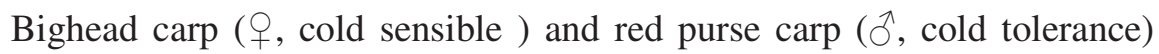
were selected to be the parents of experimental fish. Considering that bighead carp cannot survive safely while red purse carp can survive in winter, segregation in $\mathrm{F}_{2}$ of the crosses may exhibit cold tolerant and cold sensitive respectively. Therefore, $F_{2}$ was chosen as the experimental fish system in this study.

\subsection{Cold acclimation of experimental fish}

The hybrids of $\mathrm{F}_{2}$ weigned ranging from 58-70mg were maintained in a temperature-controlling aquarium at $16 \square$ for one week. Then water temperature was decreased to $10 \square$ and $4 \square$ at a rate of $2 \square$ per hour, and maintained for 5 days separately (Fig. 1). Brain tissues were collected from 5 fish of each temperature-controlling point under $10 \square$ and $4 \square$ and stored in liquid nitrogen quickly.

\subsection{RNA preparation}

Brain tissues were ground with a mortal and pestle, and then homogenized in Trizol reagent (Invitrogen Life Technologies, Carlsbad, CA). Total RNA was extracted according to the manufacturer's instructions. The cDNA of cold-treated tissues was synthesized by long distance PCR method using SMART PCR cDNA Synthesis Kit (Clontech). 


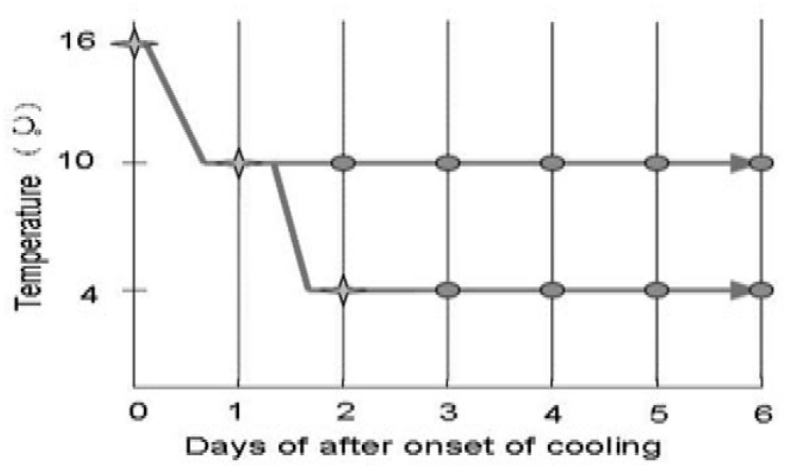

Fig. 1. Schematic diagram showing the cooling time course and sampling regime used Notes: Samples collected from temperature-controlling point represented by solid asterisk

\subsection{Construction of subtracted cDNA library}

The mixed brain samples from $10 \square$ and $4 \square$ were selected to be the object of study. SSH was carried out using Clontech PCR-Select cDNA Subtraction Kit and Advantage Klen-Taq Polymerase Mix (Clontech). One microgram each of prepared tester and driver ds cDNA were digested by Rsa $\square$ enzyme,and the tester cDNA was separated into half parts and then each was ligated to adaptor 1 and adaptor $2 \mathrm{R}$ in separated ligation reaction mixture at $16 \square$ overnight. The reaction was stopped by EDTA/glycogen and ligase was inactivated by heating the sample at $72 \square$ for $5 \mathrm{~min}$. For the first hybridization, an excess amount of driver cDNA was added to each tester cDNA (ligated with adaptor 1 and 2R) in separate sample. After denaturation at $98 \square$ for $1.5 \mathrm{~min}$, the first hybridization was performed in a hybridization buffer at $68 \square$ for $8 \mathrm{~h}$. The two samples from the first hybridization were mixed and fresh denatured driver cDNA were added. In the second hybridization, new bybrid molecules corresponding to differentially expressed cDNA with different adaptors on each end were formed. Two PCR reactions were performed using different primers to selectively amplify the differentially expressed sequences. The PCR products were cloned into pMD 18 T-vector (Takara). The subtracted cDNA library was constructed after transformation into DH5a Escherichia coli strain.

\subsection{Screening of differentially expressed clones by dot blotting}

After purification by phenol-chloroform extraction, PCR products of forward- and reverse-subtracted cDNAs were used to be the probes labeled by $\alpha-{ }^{32} \mathrm{P}$ dATP separately. 480 clones were selected at random from the 
forward subtracted library enriched for cold-related sequences and the cDNA inserts were amplified. The PCR products were then blotted onto membranes together with control cDNAs and probed with the forward and reverse subtracted cDNA pools. 26 of positive clones screened were sequenced and performed alignment in the GenBank/EMBL database using blastn searching engine.

\subsection{Real-time PCR}

Another group of fish produced through inducing the gynogenesis of bighead carp was conducted the same temperature decreasing experiments described above. The purpose of this experiment is to further validate the genes isolated by SSH. Two tissues of livers and intestines were sampled and prepared single strand cDNA from three temperature points of $16^{\circ} \mathrm{C}$, $10^{\circ} \mathrm{C}$ and $4{ }^{\circ} \mathrm{C}$ respectively. Parts of sequences obtained by SSH were used to design primer pairs with software Beacon Designer (version 4.0). Realtime PCR was carried out in the Rotor-Gene 3000 PCR (CORBETT) using

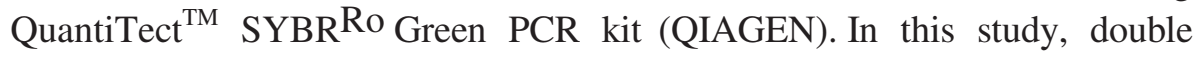
standard curves of comparative quantitation method was used to analyze the differences of gene expression at different temperature points.

\section{RESULTS AND ANALYSIS}

\subsection{Selection of the experimental fish}

In this study, the mixed brain samples from $10 \square$ and $4 \square$ were selected to be the object of study. It is reported that low temperature firstly have an effect on the central nervous system of fish. In addition, carp can live at 10$16 \square$ normally while abnormally even to death at $4 \square$. Therefore, it is hopefully to find cold-related genes through comparing these two temperature points.

\subsection{Experimental key steps: the quality of total RNA, adaptor ligation efficiency and subtracted hybridized efficiency}

Total RNA was extracted by Trizol reagents and visualized on $2 \%$ agarose gel. The value of OD260/280 was more than 2.0. It is very important to perform the ligation well before subtracted hybridization. The results show a successful ligation and subtraction using $\alpha$-tubulin gene as the control (Fig. 2). 


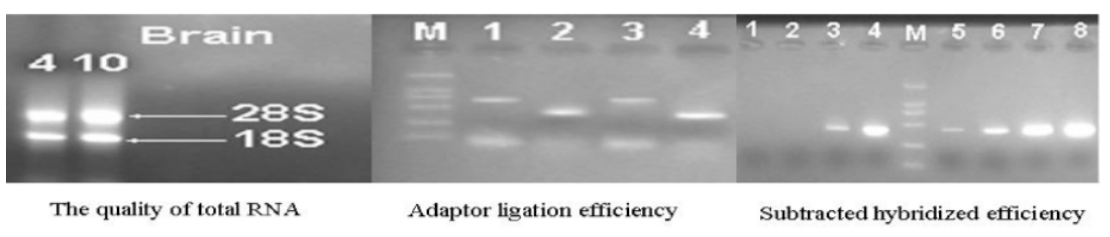

Fig. 2. The results of three key steps of this study

Notes: PCR was performed on subtracted cDNA(Lane1-4) and unsubtracted cDNA(Lane5-8); M: DNA Marker DL2000; Lane 1,5: 18 cycles; Lane 2,6: 23 cycles; Lane 3,7: 28 cycles; Lane 4,8: 33 cycles

\subsection{Dot blotting analysis}

Sixty of the 480 clones were screened positive by differential screening with forward and reverse subtracted probes and $26 \mathrm{cDNA}$ clones were selected for sequencing. As figure 3 showed, duplicate dot blots hybridized with forward $(\mathrm{A}, \mathrm{C})$ and reverse subtracted cDNA probes $(\mathrm{B}, \mathrm{D})$, respectively.
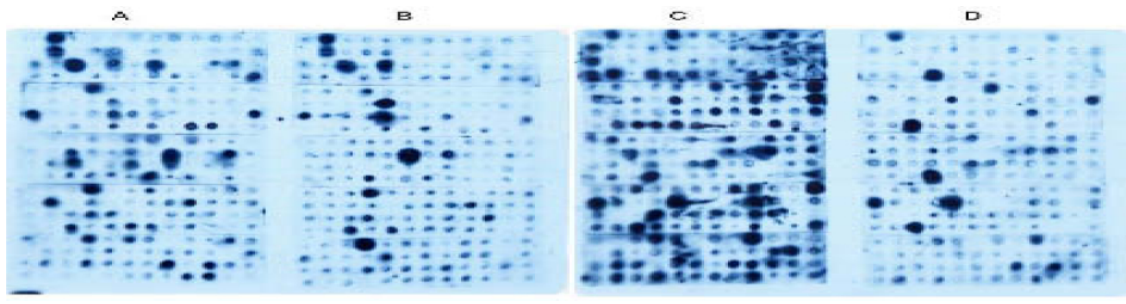

Fig. 3. Differential screening of SSH-selected cDNA clones with forward and reverse subtracted probes

Notes: A,C: probe was prepared by the forward SSH PCR $\square$ products; B,D: probe was prepared by the reverse SSH PCR $\square$ products

\subsection{Sequence analysis}

To characterize the 26 differentially expressed genes, we used blastn searching engine to find homological genes in GenBank/EMBL database. Fifteen genes show more than 85\% homology to known genes, the remaining genes are unkown function. The major categories of differentially expressed genes in this study included (1) genes for transcription factors and gene products involved in signal transduction pathways such as zinc-finger protein, brevican; (2) genes involved in lipid metabolism such as Acyl-CoA synthetases and (3) genes involved in the translational machinery such as cytochrome c oxidase, ependymin glcoprotein. 


\subsection{Validation of real-time PCR}

Two putative genes $\left(4^{\circ} \mathrm{C}\right)$ isolated by SSH named LKE-25 (col-t 22 ) and LKE-62 (col-t 29) were applied to amplify the target genes, 18srRNA of common carp was used to amplify the housekeeping gene (Table 1). Double standard curves of real-time PCR were constructed individually and showed that the values of relative coefficients $(\mathrm{R})$ are $>0.99$, most values are even beyond 0.999. Melt curves were also conducted and showed that the primer pairs are very specific in this study. The results of real-time PCR demonstrated that the concentration of LKE-25 decreases generally with the temperature decreased in different tissues, while, the concentration of LKE62 is on the rise in livers and intestines with the temperature increased. According to our results, we considered LKE-62 is a up-regulated gene and LKE-25 is a down-regulated gene at lower temperature.

Table 1. Characterization of primers for real-time PCR

\begin{tabular}{llccc}
\hline Primers & \multicolumn{1}{c}{ Primers sequence $\left(5^{\prime} \rightarrow 3^{\prime}\right)$} & Annealing temperature & Melt temperature & Cycles \\
\hline LKE-25 & $\begin{array}{l}\text { F:CATAGCCGATCAACGAACC } \\
\text { R:TAGAAACTGACCTGGATTGC }\end{array}$ & 55 & $65-95$ & 35 \\
\hline LKE-62 & $\begin{array}{l}\text { F:CTTCGTGGAGTGTGGCTAATC } \\
\text { R:CGGTTACATAGGAATGGTCTGAG }\end{array}$ & 55 & $65-95$ & 35 \\
\hline 18 srRNA & $\begin{array}{l}\text { F:CCTGTCGCCGCTGAATACC } \\
\text { R:TCGCTTTCGTCCGTCTTGC }\end{array}$ & 55 & $65-95$ & 35 \\
\hline
\end{tabular}

\section{DISCUSSIONS}

There are many studies reported the influences of low temperature in common carp, but mainly focued on the changes of membrane fluidity, lipid composition and enzyme activities. Brain is the most important organ during cold acclimation that can sense temperature and make instructions. Thus, it is necessary to do some research on the response to low temperature in the brain. By cold acclimation, the concentration of unsaturated fatty acid in the membrane increases, thereby promotes membrane fluidity (Roy et al., 1997). In addition, it is reported that ependymin (EPD) expressed increasingly in the brain and play an important role in fish to cold acclimation at an early stage. Up to now, a system analysis of differentially expressed genes in the brain is not available.

In the present study, some cold-induced genes in the brain of common carp were examined using SSH and validated by real-time PCR. It is resulted that $12.5 \%$ clones of the subtracted library showed differentially expression at low temperature. Among 26 differentially expressed genes, fifteen genes 
showed more than $85 \%$ homology to known genes while the remaining were still unknown. Because of the large numbers of unknown genes involved in cold acclimation, it is not yet possible to draw a clear picture of the molecular events that lead to adaptation or tolerance to adverse environmental conditions. Ju et al. (2002) hypothesized that a number of molecular events must occur to prepare the organism for environmental stresses including a cascade of signal transduction, activation of transcriptional factors that direct synthesis of new proteins to cope with low temperature. Many of these molecular events are rapid and transitory, but some of them may be persistently induced.

Brevican is a brain-specific proteoglycan that has been suggested to play a role in central nervous system fiber tract development (Gary et al., 1998). Besides preventing the formation of new synapses, it has been speculated that brevican might function as an insulator, sealing the synapses and preventing loss of transmitter substances from the synaptic cleft to the periphery. As the decline of temperature, brevican expressed up regulated that may prevent the formation of new synapses and lead to the low immunity of organisms. Arnab et al. (2004) isolated an intronless gene from rice encoding a zinc-finger protein and found this gene over-expressed after cold stress. However, here we characterized zinc-finger protein down regulated. It is necessary to make a further study.

Recent studies suggest that the long-chain acyl-CoA synthetases (ACS) may play a role in channeling fatty acids either toward complex lipid synthesis and storage or toward oxidation. Acyl-CoA binding protein (ACBP) was involved in fatty acid elongation and membrane assembly and organization (Gaigg et al., 2001). The detection of this gene showed that there must be some changes of cell membrane exposure to low temperature.

As for the genes involved in the translational machinery, several studies have shown that acclimation to low temperature provokes a compensatory increase of cytochrome c oxidase activity in fish tissues (Battersby et al., 1998; Thillart \& Modderkolk, 1978; Wodtke, 1981). Ependymin encoding glycoprotein (EPN) is probably associated with collagen fibrils and has the capacity to bind calcium and results in a conformational transition. It is speculated by Tang et al. (1999) that EPN plays an important role in the cold acclimation of fish. The increase of EPN under cold stress not only prevents the nerve system from damage but also promotes its regeneration.

\section{ACKNOWLEDGEMENTS}

We would like to thank Zhang QT for his hard work in this research. This work was supported by grants 2004CB117404 from the National Key Basic Research and Development Programs, Republic of China. 


\section{REFERENCES}

Arnab M, et al. Overexpression of a zinc-finger protein gene from rice confers tolerance to cold, dehydration, and salt stress in transgenic tobacco. PNAS, 2004, 101:6309-6314

Baldwin J, Hochachka PW. Functional significance of isoenzymes in thermal acclimatizationacetylcholinesterase fromtrout brain, Bioch J, 1970, 116:883-887

Battersby BJ, et al. Influence of acclimation temperature on mitochondrial DNA, RNA, and enzymes in skeletal muscle. Am. J. Physiol., 1998, 275:R905-R912

Beattie JH, et al. Cold-induced expression of the metallothionein-1 gene in brown adipose tissue of rats. Am. J. Physoil., 1996, 270:R971-R977

Brown WD. Glucose metabolism in carp. J. Cellular Comp. Physiol., 1960, 55:81-85

Cossins AR, et al. The role of desaturases in cold-induced lipid restructing. Biochem Soc. Trans., 2002, 30(6):1082-1086

Fraser NHC, et al. Temperature-dependent switch between diurnal and nocturnal foraging in salmon. Proc. R. Soc., 1993, 252:135-139

Gaigg B, et al. Depletion of acyl-coenzyme A-binding protein affects sphingolipid systhesis and causes vesicle accumulation and membrane defects in Saccharonmyces cerevisiae. Mol. Biol. Cell., 2001, 12:1147-1160

Gary SC, et al. BEHAB/brevican: a brain-specific lectican implicated in gliomas and glial cell motility. Curr. Opin. Neurobiol., 1998, 8(5):576-581

Hardewig I, et al. Temperature-dependent expression of cytochrome-c oxidase in Antarctic and temperate fish. Am. J. Physiol., 1999, 277:R508-R516

$\mathrm{Ju} Z$, et al. Differential gene expression in the brain of channel catfish (Ictalurus punctatus) in response to cold acclimation. Mol. Genet Genomics., 2002, 268:87-95

Kloepper-Sams PJ, Stegeman JJ. Effects of temperature acclimation on the expression of hepatic cytochrome P4501A mRNA and protein in the fish Fundulus heteroclitus. Arch. Biochem. Biophys., 1992, 99:38-46

Pickett MH, et al. Seasonal variation in the level of antifreeze protein mRNA from the winter flounder. Biochim. Biophys. Acta., 1983, 739:97-104

Poli A, et al. Neurochemical changes in cerebellum of goldfish exposed to various temperatures. Neurochem. Res., 1997, 22:141-149

Rodnick KJ, Sidell BD. Cold acclimation increases carnitine palmitoyltransferase activity in oxidative muscle of striped bass. Am. J. Physiol., 1994, 266:R405-R412

Roussel D, et al. Increase in the adenine nucleotide translocase content of ducking subsarcolemmal mitochondria during cold acclimation. FEBS Letters, 2000, 477:141-144

Roy R, et al. Regulation of membrane lipid bilayer structure during seasonal variation: a study on the brain membranes of Clarias batrachus. Biochim. Biophys. Acta., 1997, 1323(1):65-74

Tang SJ, et al. Cold-induced ependymin expression in zebrafish and carp brain: implications for cold acclimation. FEBS Letters, 1999, 459:95-99

Thillart V, Modderkolk J. The effect of acclimation temperature on the activation energies of state III respiration and the unsaturation of membrane lipids of gold fish mitochondria. Biochim. Biophys. Acta., 1978, 510:38-51

Tiku PE, et al. Cold-inducible expression of desaturase by transcriptional an post-translational mechanisms. Science, 1996, 271:815-818

Wodtke E. Temperature adaptation of biological membranes. Compensation of the molar activity of cytochrome $\mathrm{c}$ oxidase in the mitochondrial energy-transducing membrane during thermal acclimation of the carp (Cyprinus carpio L.). Biochim. Biophys. Acta., 1981, 640:710-720

Yeo YK, et al. Ether lipid composition and molecular species alterations in carp brain (Cyprinus carpio L.) during normoxic temperature acclimation. Neurochem. Res., 1997, 22(10):57-64 